\title{
Are people with metabolically healthy obesity really healthy? A prospective cohort study of 381,363 UK Biobank participants
}

\author{
Ziyi Zhou ${ }^{1}$ • John Macpherson ${ }^{1} \cdot$ Stuart R. Gray ${ }^{2}$ - Jason M. R. Gill ${ }^{2}$ Paul Welsh ${ }^{2}$ - Carlos Celis-Morales ${ }^{1,2,3}$. \\ Naveed Sattar ${ }^{2} \cdot$ Jill P. Pell ${ }^{1}$. Frederick K. Ho ${ }^{1}$
}

Received: 11 January 2021 / Accepted: 19 March 2021 / Published online: 10 June 2021

(C) The Author(s) 2021

\begin{abstract}
Aims/hypothesis People with obesity and a normal metabolic profile are sometimes referred to as having 'metabolically healthy obesity' (MHO). However, whether this group of individuals are actually 'healthy' is uncertain. This study aims to examine the associations of MHO with a wide range of obesity-related outcomes.

Methods This is a population-based prospective cohort study of 381,363 UK Biobank participants with a median follow-up of 11.2 years. $\mathrm{MHO}$ was defined as having a $\mathrm{BMI} \geq 30 \mathrm{~kg} / \mathrm{m}^{2}$ and at least four of the six metabolically healthy criteria. Outcomes included incident diabetes and incident and fatal atherosclerotic CVD (ASCVD), heart failure (HF) and respiratory diseases.

Results Compared with people who were not obese at baseline, those with MHO had higher incident HF (HR 1.60; 95\% CI 1.45, 1.75 ) and respiratory disease (HR 1.20; 95\% CI 1.16, 1.25) rates, but not higher ASCVD. The associations of MHO were generally weaker for fatal outcomes and only significant for all-cause (HR 1.12; 95\% CI 1.04, 1.21) and HF mortality rates (HR $1.44 ; 95 \%$ CI 1.09, 1.89). However, when compared with people who were metabolically healthy without obesity, participants with MHO had higher rates of incident diabetes (HR 4.32; 95\% CI 3.83, 4.89), ASCVD (HR 1.18; 95\% CI 1.10, 1.27), HF (HR 1.76 ; $95 \%$ CI 1.61, 1.92), respiratory diseases (HR 1.28; 95\% CI 1.24, 1.33) and all-cause mortality (HR 1.22; 95\% CI 1.14, 1.31). The results with a 5 year landmark analysis were similar.

Conclusions/interpretation Weight management should be recommended to all people with obesity, irrespective of their metabolic status, to lower risk of diabetes, ASCVD, HF and respiratory diseases. The term 'MHO' should be avoided as it is misleading and different strategies for risk stratification should be explored.
\end{abstract}

Keywords Cardiovascular disease $\cdot$ Metabolically healthy obesity $\cdot$ Obesity

\begin{abstract}
Abbreviations
ASCVD Atherosclerotic CVD

COPD Chronic obstructive pulmonary disease

$\mathrm{HF} \quad$ Heart failure

$\overline{\text { Carlos Celis-Morales, Naveed Sattar, Jill P. Pell and Frederick K. Ho are }}$ joint senior authors.
\end{abstract}

Frederick K. Ho

Frederick.Ho@glasgow.ac.uk

1 Institute of Health and Wellbeing, University of Glasgow, Glasgow, UK

2 Institute of Cardiovascular and Medical Sciences, University of Glasgow, Glasgow, UK

3 Human Performance Laboratory, Education, Physical Activity and Health Research Unit, University Católica del Maule, Talca, Chile

$\begin{array}{ll}\text { MHN } & \text { Metabolically healthy non-obesity } \\ \text { MHO } & \text { Metabolically healthy obesity } \\ \text { MI } & \text { Myocardial infarction } \\ \text { MUN } & \text { Metabolically unhealthy non-obesity } \\ \text { MUO } & \text { Metabolically unhealthy obesity }\end{array}$

\section{Introduction}

Over 300 million people are estimated to be obese worldwide [1]. Based on current trends, it is estimated there will be 1 billion obese people by 2030 , accounting for $20 \%$ of the world's adult population $[1,2]$. Obesity is the main cause of the current global epidemics of type 2 diabetes, hypertension, CVD and many other diseases [3, 4]. 


\section{Research in context}

\section{What is already known about this subject?}

- Individuals who are obese and with a normal metabolic profile are sometimes referred to as having 'metabolically healthy obesity' (MHO)

- Compared with individuals who are not obese with a normal metabolic profile, those with MHO have higher risk of diabetes and atherosclerotic CVD (ASCVD)

\section{What is the key question?}

- Are people with $\mathrm{MHO}$ also at a higher risk of other obesity-related outcomes, such as heart failure and respiratory diseases?

\section{What are the new findings?}

- Compared with people who were metabolically healthy without obesity, people with MHO were at a higher risk of all-cause mortality, incident diabetes, ASCVD, heart failure and respiratory disease, although the risk was lower compared with people with obesity and an abnormal metabolic profile

- Among people with $\mathrm{MHO}$ at baseline who remained obese, over one-third became metabolically unhealthy within 3 to 5 years. These people had an even higher risk of ASCVD

\section{How might this impact on clinical practice in the foreseeable future?}

- Weight management should be recommended to all people with obesity, irrespective of their metabolic status; the term 'metabolically healthy obesity' should be avoided as it is misleading

Typically, obesity leads to metabolic dysfunctions such as elevated blood glucose, increased BP, dyslipidaemia (characterised by high triacylglycerols and lower HDLcholesterol levels), systemic inflammation and insulin resistance [5]. However, some people with obesity have normal $\mathrm{BP}$, favourable lipid and inflammatory profiles, and high insulin sensitivity $[6,7]$. This phenotype is sometimes referred to as 'metabolically healthy obesity' (MHO) $[6,7]$. The prevalence of $\mathrm{MHO}$ is estimated to be $3 \%$ to $22 \%$ in the general population $[8,9]$.

People with MHO have been shown to have an elevated risk of diabetes [10], but the evidence on atherosclerotic CVD (ASCVD) and all-cause mortality is mixed [11-13]. There are multiple reasons behind these mixed results. First, the definitions of $\mathrm{MHO}$ were inconsistent across studies [14]. Some studies defined MHO as participants who fulfil almost all of the criteria, resulting in a lower prevalence of $\mathrm{MHO}$ with similar risk to that in the general non-obese population [14]. It has been suggested that MHO may be a transitional state, rather than a distinct and stable phenotype $[15,16]$, and that people with $\mathrm{MHO}$ eventually develop metabolic dysfunction known as metabolically unhealthy obesity (MUO) [15]. At this stage, they have a higher risk of ASCVD and all-cause mortality, similar to those who originally had MUO. Importantly, research so far has focused on the association of MHO with ischaemic heart disease, stroke, diabetes and all-cause mortality, but has omitted other important obesityrelated conditions such as respiratory diseases or heart failure
(HF) [14]. In addition, even though diabetes is often thought of as a mediator between obesity and cardiovascular outcomes, there has been no study examining the mechanistic role of diabetes between $\mathrm{MHO}$ and CVD.

This study aimed to address the limitations of earlier research and determine the association of $\mathrm{MHO}$, as well as its transition, with all-cause mortality, diabetes, ASCVD, HF and respiratory diseases. This study further explored to what extent diabetes mediates the association between $\mathrm{MHO}$ and cardiovascular outcomes.

\section{Methods}

Study design and participants UK Biobank is a prospective cohort study. Between 2007 and 2010, UK Biobank recruited 502,493 participants from the general population [17]. Participants attended one of 22 assessment centres across England, Scotland and Wales where they completed a selfadministered, touch-screen questionnaire and face-to-face interview, and trained staff took a series of measurements including height, weight and BP. This study included only the 381,363 participants who were not underweight and had complete data on height, weight, BP and blood-based biomarkers (Fig. 1). The analysis of the transition of metabolic status included the subgroup of 8521 participants who had complete re-assessment of their metabolic status at median follow-up of 4.4 (IQR 3.7-4.9) years. 
Fig. 1 Participant flowchart

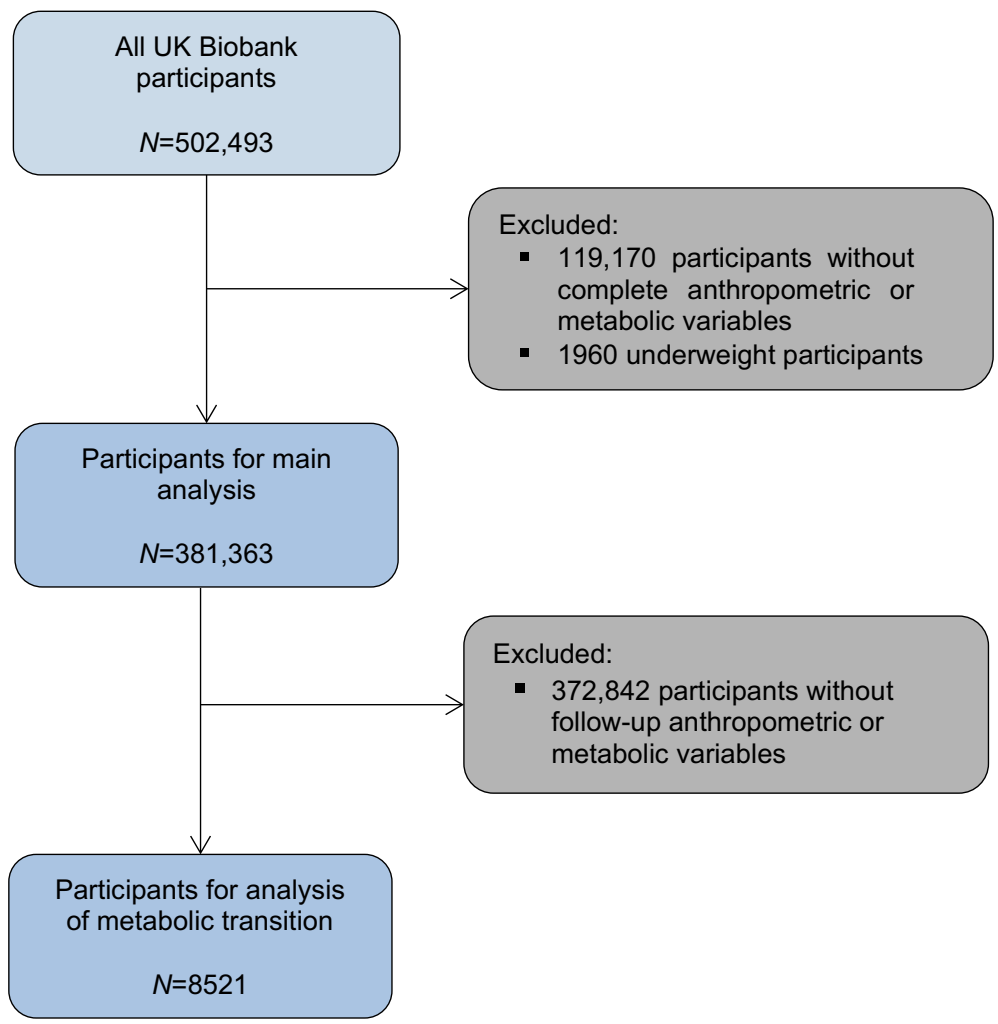

Obesity Height was measured to the nearest centimetre, using a Seca 202 stadiometer (Hamburg, Germany), and body weight to the nearest $0.1 \mathrm{~kg}$, using a Tanita BC-418 body composition analyser (Tokyo, Japan). BMI was calculated as weight/height ${ }^{2}$. Participants with $\mathrm{BMI} \geq 30 \mathrm{~kg} / \mathrm{m}^{2}$ were classified as obese.

Metabolic status Our analyses included six metabolic markers to define metabolic health as a binary condition, including BP and five blood-based biomarkers: C-reactive protein (CRP), triacylglycerols, LDL-cholesterol, HDL-cholesterol and $\mathrm{HbA}_{1 \mathrm{c}}$. BP was measured by a nurse using an automated machine (or manually if unavailable), and the mean of available measurements was derived. Serum biomarkers were measured at a dedicated central laboratory between 2014 and 2017. These measures were externally quality assured and were quality controlled [18]. The cut-off values to define metabolically healthy were adapted from a previous study [19] and are shown in Electronic supplementary material (ESM) Table 1. Participants who fulfilled at least four (out of six) metabolically healthy criteria were considered metabolically healthy.

Metabolic health and obesity Based on BMI and metabolic status, participants were categorised as having: metabolically healthy non-obesity (MHN, reference group), MHO, metabolically unhealthy non-obesity (MUN) or MUO. In a sensitivity analysis, MHN and MUN were combined as non-obesity.
Outcomes Outcomes were ascertained through individuallevel record linkage of the UK Biobank cohort to routine administrative databases. Date and cause of death were obtained from death certificates held by the National Health Service Information Centre (England and Wales) and the National Health Service Central Register (Scotland). Dates and causes of hospital admissions were obtained through record linkage to Health Episode Statistics (England and Wales) and Scottish Morbidity Records (Scotland). Detailed information about the linkage procedures can be found at http://content.digital.nhs. $\mathrm{uk} /$ services. Incident diabetes was ascertained through linkage to the primary care data, which are available in about $45 \%$ of all participants. Primary care data were used to ascertain diabetes because patients with diabetes are routinely managed in general practitioner practices in the UK. At the time of analysis, mortality data were available up to 30 June 2020, hospital admission data were available up to 31 May 2020 for participants in England and 31 March 2017 for those in Scotland and Wales, and primary care data were available up to 2016 to 2017. We defined diabetes as type 2 diabetes mellitus (ICD-10 code E11), and ASCVD as fatal ischaemic heart disease (I10-25), or non-fatal myocardial infarction (MI) (I21), or fatal/non-fatal stroke (I60-64) per the American College of Cardiology definition [20]. In addition, HF was defined as I11.0, I42.0, I42.6-42.7, I42.9 and I50; chronic respiratory diseases as I26-28 and J30-99; and chronic obstructive pulmonary disease (COPD) as J41-44. 
Covariates Ethnicity, highest level of education, television viewing time, smoking status, alcohol consumption and dietary intake were self-reported. Physical activity was selfreported using the validated International Physical Activity Questionnaire [21]. Townsend area deprivation index was obtained from postcode of residence and is derived using aggregated data on unemployment, car and home ownership, and household overcrowding [22]. Baseline prevalent conditions were self-reported in a nurse-led interview.

Statistical analyses Means and SDs were used to describe continuous variables. Cox proportional hazards models were used to analyse the associations between MHO and health outcomes, with the results reported as HRs and 95\% CIs. The models were adjusted for age, sex and ethnicity in Model 1; and additionally for education level and deprivation index in Model 2; and additionally for smoking, alcohol drinking, television viewing, physical activity and intake of fruit and vegetables, oily fish, red meat and processed meat in Model 3. These factors were chosen as they are plausible confounders, based on prior knowledge.

The dose-response relationships of BMI and metabolic status with health outcomes were analysed using a penalised cubic spline in a Cox model, adjusting for the same covariates, with four metabolically healthy criteria as the reference points. Penalised splines are a variation of basis splines and are robust against the number of knots and knot placements [23]. The interactions between BMI and metabolic status were tested by using likelihood ratio tests.

Two sensitivity analyses were conducted. First, nonobesity (whether MHN or MUN) was used as the reference group to examine the risk of MHO compared with all nonobese participants, instead of MHN. Second, we conducted a 5 year landmark analysis, excluding any participant who developed the outcome of interest in the first 5 years of follow-up to mitigate the potential effect of reverse causation.

Participants with longitudinal BMI and metabolic data were categorised as MHN throughout, $\mathrm{MHO}$ throughout, transition from MHO to MUO, or MUO throughout, which covers the most common scenarios. These four categories were then used as a primary exposure variable in Cox models. Participants not in any of the four categories were excluded in the transition analysis as there were not sufficient numbers of events to support reliable inference. Proportional hazards assumptions were checked using Schoenfeld residuals.

The mechanistic role of diabetes between MHO and outcomes was explored in two steps. We first examined whether incident diabetes was still a significant predictor of outcomes after adjusting for $\mathrm{MHO}$ and other covariates. If so, formal mediation analyses using the counterfactual-based mediation framework were conducted [24]. All analyses were conducted using R version 4.0.3 with the package survival (https://www.r-project.org/ accessed 11 October 2020).
Ethics approval UK Biobank received ethics approval from the North West Multi-Centre Research Ethics Committee (REC reference: 11/NW/03820). All participants gave written informed consent before enrolment in the study, which was conducted in accordance with the principles of the Declaration of Helsinki. Direct dissemination of the results to participants is not possible/applicable.

\section{Results}

There were 381,363 participants included in the main analysis (Fig. 1), of which 208,625 (54.7\%) had MHN, 35,103 (9.2\%) MHO, 78,259 (20.5\%) MUN and 59,376 (15.6\%) MUO. Participants with MUO at baseline, compared with MHN, were older, watched more television, exercised less, had lower education level, higher deprivation index and higher red and processed meat intake, were more likely to be male and of non-white ethnicity, and were less likely to be a current smoker (Table 1). Compared with MUO, people with MHO were younger, watched less television, exercised more, had higher education level, lower deprivation index and higher red and processed meat intake, and were less likely to be male and of non-white ethnicity.

The median (IQR) follow-up period was 11.2 (10.3-11.9) years. In Model 3, compared with participants with MHN at baseline, those with $\mathrm{MHO}$ had higher rates of incident diabetes (HR 4.32; 95\% CI 3.83, 4.89) (Fig. 2), ASCVD (HR 1.18; 95\% CI 1.10, 1.27), MI (HR 1.23; 95\% CI 1.11, 1.37), stroke (HR 1.10; 95\% CI 1.01, 1.21), HF (HR 1.76; 95\% CI 1.61, 1.92), respiratory diseases (HR 1.28; 95\% CI 1.24, 1.33) and COPD (HR 1.19; 95\% CI 1.11, 1.28) (Fig. 3). Generally, rates of cardiovascular and respiratory outcomes were highest in MUO, followed by MUN and MHO, except for incident and fatal $\mathrm{HF}$, and incident respiratory diseases. In these outcomes, people with MHO had higher rates than those with MUN. The associations between $\mathrm{MHO}$ and mortality outcomes were generally similar, except that MHO was not significantly associated with stroke or COPD mortality. Participants with MHO had higher all-cause mortality rates (HR 1.22; 95\% CI 1.14, 1.31) compared with participants with MHN.

Compared with participants without obesity (regardless of MHN or MUN) at baseline, those with MHO had higher rates of diabetes (HR 2.06; 95\% CI 1.77, 2.40), HF (HR 1.60; 95\% CI $1.45,1.75)$ and respiratory diseases (HR $1.20 ; 95 \%$ CI $1.16,1.25$ ), but not ASCVD, including MI and stroke (ESM Fig. 1). The associations of MHO were generally weaker for fatal outcomes and only significant for all-cause mortality (HR 1.12; 95\% CI 1.04, 1.21) and HF mortality rates (HR 1.44; 95\% CI 1.09, 1.89). The results for Models 1 and 2 are shown in ESM Tables 2 and 3. The results of the 5 year landmark analysis were similar (ESM Fig. 2). 
Table 1 Participant characteristics

\begin{tabular}{|c|c|c|c|c|}
\hline Characteristic & MHN & $\mathrm{MHO}$ & MUN & MUO \\
\hline Total $N$ & $208,625(54.71)$ & $35,103(9.20)$ & $78,259(20.52)$ & $59,376(15.57)$ \\
\hline Male & $84,648(40.57)$ & $15,166(43.20)$ & $47,292(60.43)$ & $30,591(51.52)$ \\
\hline Age, years, mean (SD) & $55.80(8.19)$ & $56.04(8.11)$ & $58.52(7.68)$ & $57.53(7.67)$ \\
\hline \multicolumn{5}{|l|}{ Ethnicity } \\
\hline White & $198,496(95.55)$ & $32,817(93.98)$ & $73,060(93.79)$ & $55,913(94.70)$ \\
\hline South Asian & $1243(0.60)$ & $230(0.66)$ & $372(0.48)$ & $333(0.56)$ \\
\hline Black & $3183(1.53)$ & $454(1.30)$ & $2617(3.36)$ & $1147(1.94)$ \\
\hline Chinese & $2356(1.13)$ & $1022(2.93)$ & $808(1.04)$ & $1091(1.85)$ \\
\hline Mixed & $803(0.39)$ & $27(0.08)$ & $286(0.37)$ & $35(0.06)$ \\
\hline Other & $1649(0.79)$ & $370(1.06)$ & $753(0.97)$ & $524(0.89)$ \\
\hline \multicolumn{5}{|l|}{ Education level } \\
\hline College or university degree & $77,114(36.99)$ & $9475(27.03)$ & $21,896(28.01)$ & $13,294(22.42)$ \\
\hline A levels/AS levels or equivalent & $24,610(11.81)$ & $3747(10.69)$ & $7995(10.23)$ & $5956(10.04)$ \\
\hline O levels/GCSE or equivalent & $44,157(21.18)$ & $7913(22.57)$ & $16,569(21.19)$ & $12,870(21.70)$ \\
\hline CSEs or equivalent & $10,932(5.24)$ & $2396(6.84)$ & $3926(5.02)$ & $3687(6.22)$ \\
\hline NVQ or HND or HNC or equivalent & $11,809(5.67)$ & $2579(7.36)$ & $5936(7.59)$ & $4912(8.28)$ \\
\hline Other professional qualifications & $10,216(4.90)$ & $1895(5.41)$ & $4268(5.46)$ & $3410(5.75)$ \\
\hline Prefer not to answer & $1813(0.87)$ & $434(1.24)$ & $1037(1.33)$ & $852(1.44)$ \\
\hline None of the above & $27,795(13.33)$ & $6615(18.87)$ & $16,558(21.18)$ & $14,326(24.16)$ \\
\hline Deprivation index, mean (SD) & $-1.55(2.94)$ & $-1.03(3.17)$ & $-1.32(3.09)$ & $-0.78(3.26)$ \\
\hline \multicolumn{5}{|l|}{ Smoking } \\
\hline Never & $120,690(58.10)$ & $18,931(54.24)$ & $38,372(49.29)$ & $28,746(48.74)$ \\
\hline Previous & $67,773(32.63)$ & $13,183(37.77)$ & $28,197(36.22)$ & $23,944(40.60)$ \\
\hline Current & $19,257(9.27)$ & $2787(7.99)$ & $11,285(14.50)$ & $6292(10.67)$ \\
\hline Alcohol consumption, units/week, mean (SD) & $15.81(17.24)$ & $16.78(20.17)$ & $17.94(20.78)$ & $16.45(21.26)$ \\
\hline TV viewing, hours/day, mean (SD) & $2.54(1.46)$ & $3.05(1.58)$ & $3.00(1.61)$ & $3.39(1.74)$ \\
\hline Physical activity, MET min/week, mean (SD) & $2773.06(2249.72)$ & $2530.02(2166.41)$ & $2629.98(2212.36)$ & $2362.97(2073.92)$ \\
\hline Fruit/vegetable intake, portions/week, mean (SD) & $4.21(2.41)$ & $4.16(2.52)$ & $3.91(2.45)$ & $4.00(2.47)$ \\
\hline \multicolumn{5}{|l|}{ Oily fish intake } \\
\hline Never & $20,250(9.76)$ & $3972(11.40)$ & $9197(11.85)$ & $7750(13.18)$ \\
\hline Less than once a week & $67,696(32.63)$ & $11,419(32.79)$ & $26,165(33.71)$ & $20,092(34.17)$ \\
\hline Once a week & $80,672(38.89)$ & $12,841(36.87)$ & $28,952(37.30)$ & $21,102(35.89)$ \\
\hline 2-4 times a week & $36,758(17.72)$ & $6222(17.87)$ & $12,632(16.28)$ & $9356(15.91)$ \\
\hline 5-6 times a week & $1616(0.78)$ & $271(0.78)$ & $497(0.64)$ & $344(0.59)$ \\
\hline Once or more daily & $471(0.23)$ & $102(0.29)$ & $170(0.22)$ & $153(0.26)$ \\
\hline Red meat intake, portions/week, mean (SD) & $2.00(1.38)$ & $2.21(1.50)$ & $2.18(1.48)$ & $2.32(1.57)$ \\
\hline \multicolumn{5}{|l|}{ Processed meat intake } \\
\hline Never & $22,931(11.02)$ & $2431(6.95)$ & $5898(7.56)$ & $3268(5.53)$ \\
\hline Less than once a week & $68,018(32.70)$ & $10,355(29.62)$ & $21,258(27.26)$ & $15,105(25.55)$ \\
\hline Once a week & $59,829(28.76)$ & $10,387(29.71)$ & $23,265(29.84)$ & $17,767(30.06)$ \\
\hline 2-4 times a week & $50,111(24.09)$ & $10,360(29.64)$ & $23,875(30.62)$ & $19,898(33.66)$ \\
\hline 5-6 times a week & $5753(2.77)$ & $1130(3.23)$ & $2884(3.70)$ & $2425(4.10)$ \\
\hline Once or more daily & $1375(0.66)$ & $293(0.84)$ & $790(1.01)$ & $647(1.09)$ \\
\hline BMI, $\mathrm{kg} / \mathrm{m}^{2}$, mean (SD) & $24.94(2.63)$ & $33.05(3.04)$ & $26.62(2.24)$ & $34.42(4.03)$ \\
\hline Number of MH factors, mean (SD) & $4.47(0.64)$ & $4.22(0.46)$ & $2.67(0.58)$ & $2.41(0.77)$ \\
\hline $\mathrm{SBP}, \mathrm{mmHg}$, mean (SD) & $134.17(18.59)$ & $138.62(18.28)$ & $144.03(16.92)$ & $144.04(17.16)$ \\
\hline DBP, mmHg, mean (SD) & $79.87(9.84)$ & $84.38(9.85)$ & $84.58(9.36)$ & $86.67(9.61)$ \\
\hline Any antihypertensive medications & $26,858(12.87)$ & $9164(26.11)$ & $22,997(29.39)$ & $25,255(42.53)$ \\
\hline CRP, mg/l, mean (SD) & $1.45(3.15)$ & $2.37(3.59)$ & $4.12(6.39)$ & $5.18(5.91)$ \\
\hline
\end{tabular}


Table 1 (continued)

\begin{tabular}{lllll}
\hline Characteristic & MHN & MHO & MUN & MUO \\
\hline Triacylglycerols, mmol/l, mean (SD) & $1.32(0.56)$ & $1.51(0.57)$ & $2.46(1.20)$ & $2.51(1.25)$ \\
LDL-cholesterol, mmol/l, mean (SD) & $3.50(0.82)$ & $3.46(0.86)$ & $3.73(0.93)$ & $3.58(0.93)$ \\
HDL-cholesterol, mmol/l, mean (SD) & $1.58(0.37)$ & $1.40(0.30)$ & $1.29(0.35)$ & $1.21(0.30)$ \\
Any cholesterol lowering medications & $22,495(10.78)$ & $6560(18.69)$ & $20,104(25.69)$ & $19,805(33.36)$ \\
$\mathrm{HbA}_{1 \mathrm{c}}, \mathrm{mmol} / \mathrm{mol}$, mean (SD) & $34.42(3.93)$ & $35.35(4.23)$ & $37.90(8.52)$ & $40.45(10.34)$ \\
$\mathrm{HbA}_{1 \mathrm{c}}, \%$, mean & 5.3 & 5.4 & 5.6 & 5.9 \\
\hline
\end{tabular}

Numbers are $n(\%)$ unless otherwise specified

Some sub-categories, such as education level, may not add up due to missing data

A levels, Advanced levels; AS levels, Advanced Subsidiary levels; CRP, C-reactive protein; CSEs, Certificate of Secondary Education; DBP, diastolic BP; GCSE, General Certificate of Secondary Education; HNC, Higher National Certificates; HND, Higher National Diplomas; MET, metabolic equivalent of tasks; MH, metabolically healthy; NVQ, National Vocational Qualification; O levels, Ordinary levels; SBP, systolic BP; TV, television

The interactions between obesity and metabolic health factors are shown in ESM Fig. 3. The associations of metabolic health factors with incident respiratory diseases $\left(p_{\text {interaction }}=0.001\right)$ were slightly stronger among people who were not obese. There were no other significant interactions. There were no significant time trends in the Schoenfeld residuals, except for diabetes, suggesting these associations were relatively stable during the follow-up (ESM Fig. 4). The trend for diabetes appeared to be increasing, suggesting the association between $\mathrm{MHO}$ and diabetes may be increasing over time, and the current HR estimate should not be extrapolated for different follow-up duration.

ESM Fig. 5 shows the transition of metabolic status among the subgroup of 8521 participants who had longitudinal BMI and metabolic data (median [IQR] follow-up 4.4 [3.7-4.9] years). Half of the participants who had MHO at baseline remained so in the follow-up, $20 \%$ became non-obese and over one-quarter transitioned to MUO. Figure 4 shows the associations between transition of MHO status and health outcomes. Compared with participants with MHN throughout, participants who transitioned from MHO to MUO had higher rates of incident ASCVD (HR 2.46; 95\% CI 1.12, 5.41) and all-cause mortality (HR 3.07; 95\% CI 1.44, 6.56). There were no significant associations for the MHOthroughout group. After controlling for baseline MHO status, incident diabetes was no longer a significant risk factor for any of the outcomes (ESM Table 4) and thus was not a mediator.

\section{Discussion}

Principal findings The current study demonstrated that people with $\mathrm{MHO}$ were at a substantially higher risk of diabetes, ASCVD, HF, respiratory diseases and all-cause mortality compared with people with MHN. Particularly worth noting is that people with $\mathrm{MHO}$ were at an even higher risk than those with MUN of HF and respiratory disease. Among people with $\mathrm{MHO}$ at baseline who remained obese, over one-third became metabolically unhealthy within 3 to 5 years. These people acquired an even higher risk of ASCVD. In addition, there were weak or non-significant interactions of obesity and metabolic health factors with health outcomes. The key point therefore is that the risk of many important outcomes, such as HF and respiratory disease, is elevated in people with obesity even if they have a normal metabolic profile. Using the label 'metabolically healthy' to describe this group in clinical medicine is misleading and therefore should be avoided.

Strengths and limitations of this study This study has several strengths over the previous evidence. First, we were able to investigate emerging outcomes such as $\mathrm{HF}$ and respiratory diseases that have often been omitted in MHO studies. In fact, the associations with these outcomes, along with the instability of the MHO status, highlight that MHO is not a healthy

Number Event $\quad$ HR $(95 \% \mathrm{Cl})$

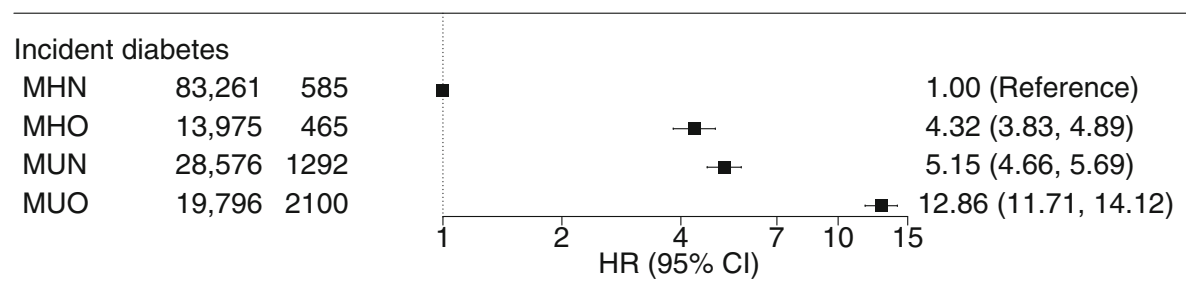

Fig. 2 Association between MHO and incident diabetes with MHN as reference group. Adjusted for each other and for age, sex, ethnicity, education, deprivation, smoking, alcohol drinking, television viewing, physical activity and intake of fruit and vegetables, oily fish, red meat and processed meat 
Fig. 3 Association between $\mathrm{MHO}$ and cardiovascular and respiratory outcomes with MHN as reference group. Adjusted for each other and for age, sex, ethnicity, education, deprivation, smoking, alcohol drinking, television viewing, physical activity, and intake of fruit and vegetables, oily fish, red meat and processed meat

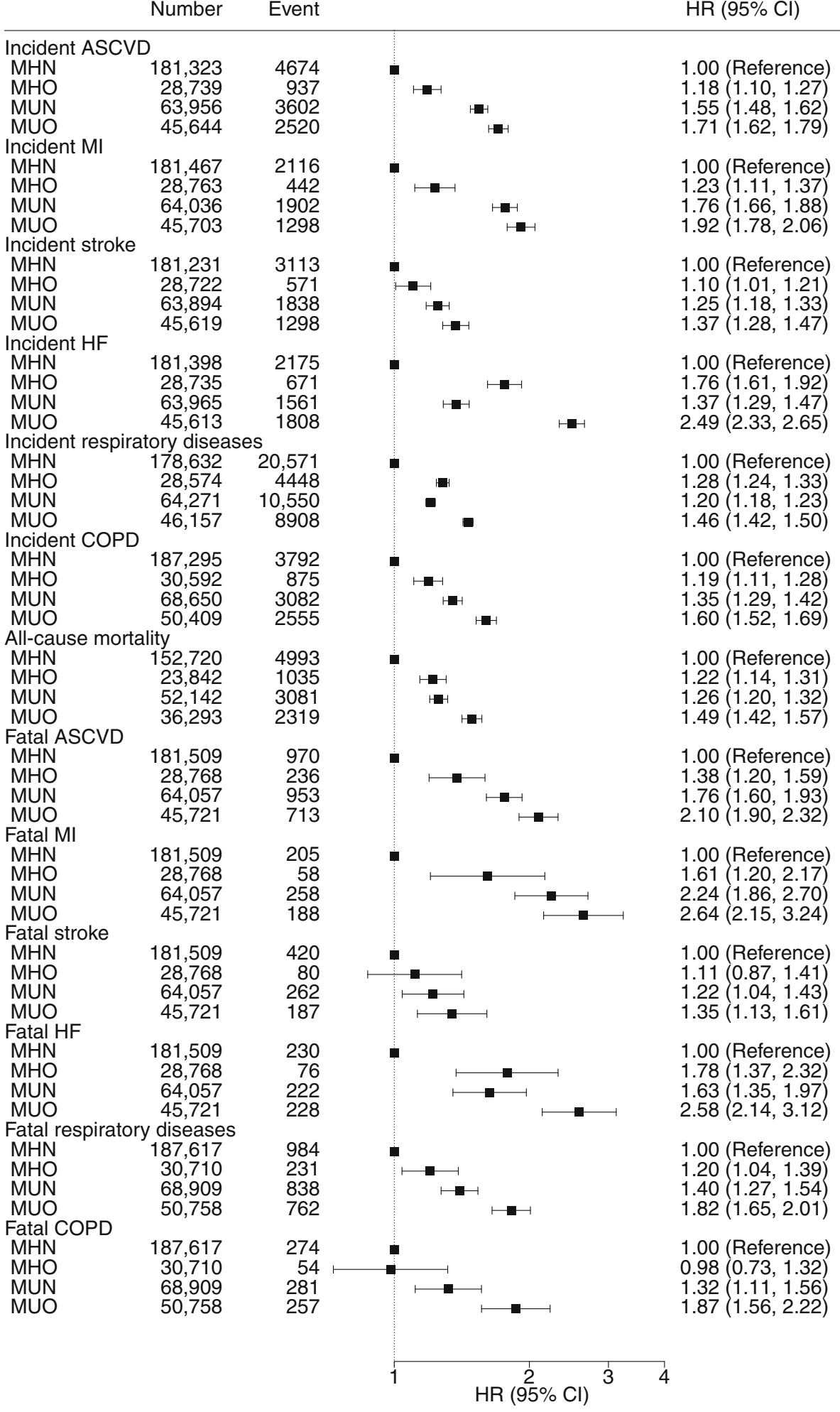

state. Furthermore, this study conducted a 5 year landmark analysis which indicated that the findings were unlikely to simply reflect reverse causation. We also adjusted for a wide range of potential confounders, including individual- and area-level socioeconomic status and lifestyle factors. Using mediation analysis, the current study also found that individuals with MHO may develop ASCVD and HF outcomes through diabetes-independent pathways. However, as with any observational study, residual confounding, such as from excess energy intake and family history of diabetes and 
Fig. 4 Association of change of metabolic status with health outcomes. Adjusted for age, sex ethnicity, education, deprivation, smoking, alcohol drinking, television viewing, physical activity, and intake of fruit and vegetables, oily fish, red meat and processed meat

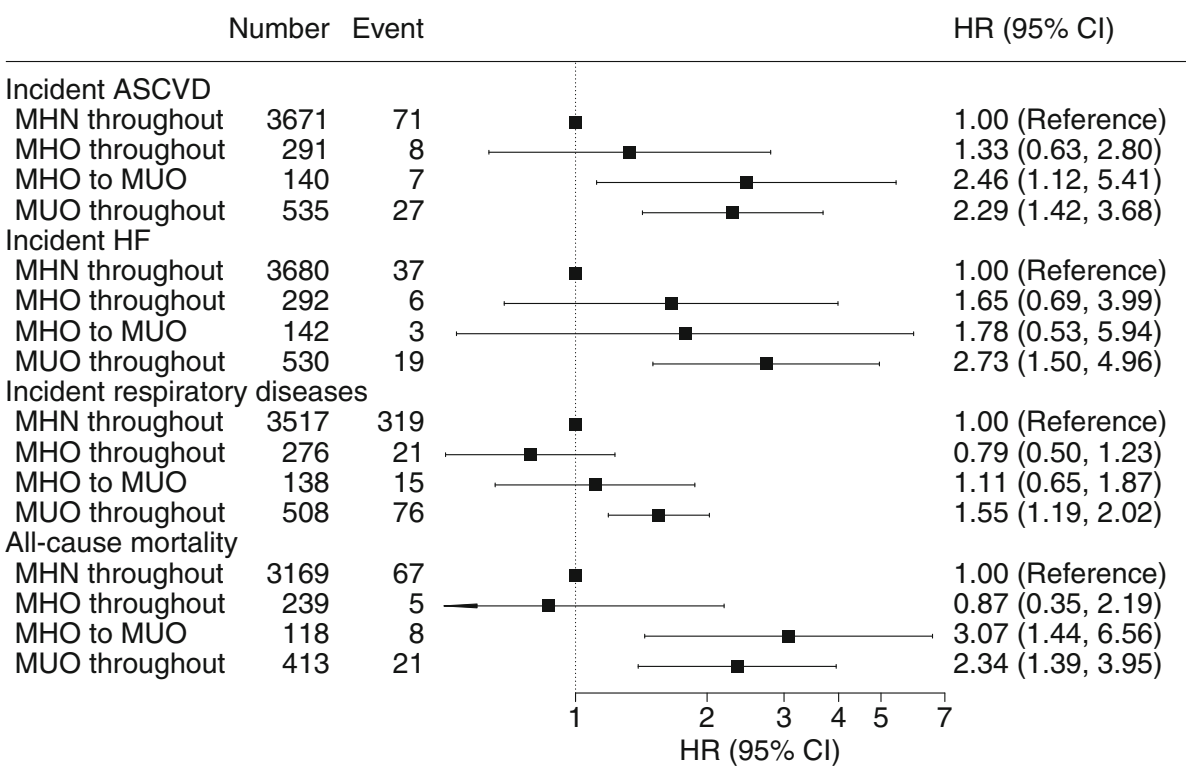

study was similar to our current study, suggesting that their lack of statistical significance could be due to insufficient power. It is worth noting that this previous literature has mostly focused on ASCVD, including ischaemic heart disease and stroke, and often omitted HF. Importantly, we found that MHO was associated with substantially higher risk of HF even compared with those with MUN. This could be related to a range of mechanistic factors beyond the usual metabolic aspect, such as haemodynamic perturbances, that likely link obesity to HF risk [31]. Notably, others have shown that obesity may be more strongly linked to incident HF than to MI [32].

To our knowledge, no previous study has directly examined the association between MHO and respiratory diseases, including COPD. However, it is well recognised that obesity is generally associated with lower respiratory function and a wide range of respiratory diseases, such as COPD, obstructive sleep apnoea and obesity hypoventilation syndrome [33]. There have been several hypotheses as to why obesity may be associated with COPD, including fat oxidative capacity, inflammation and insulin resistance [34]. However, as shown in this study, having a normal metabolic profile, and $\mathrm{HbA}_{1 \mathrm{c}}$, did not guarantee lower risk of respiratory diseases among obese people. Of note, chronic respiratory diseases account for considerable morbidity and mortality worldwide and are estimated to have been the third leading cause of death in 2017 [35].

Implications of this study Although our results suggested that people with MHO may have lower cardiovascular and respiratory risk compared with MUO, their risk was still higher than those who were metabolically healthy without obesity, especially with respect to HF and respiratory diseases. These findings, as well as the unstable nature of $\mathrm{MHO}$, suggest that 
weight management could be beneficial for people with obesity even if they do not currently show abnormalities in their metabolic profile. Weight management strategies include lifestyle changes, such as diet and physical activity, concomitant pharmacotherapies upon risk assessment $[36,37]$ or bariatric surgery in severe obesity [38].

It is worth noting that half of the participants remained with MHO after 4.4 years of follow-up. We could not detect any significant elevated risk among them compared with people who were metabolically healthy and non-obese throughout the study. It is likely that this group of people are at lower risk than people with other MHO trajectories. However, since there were not sufficient numbers of events, we cannot conclude whether they were at the same risk as people with $\mathrm{MHN}$, or were at a modestly elevated risk. Future prospective studies should consider this research question.

This study also showed that people with obesity are a heterogenous group, and there is a potential to risk stratify based on prognosis. For example, people with MUO were at a higher risk of mortality and morbidity than everyone else, and thus they should be prioritised for intervention. However, it should be noted that using a single binary label (i.e. 'MHO') for clinical management may have questionable utility. Obesity is associated with a wide range of diseases and using a single label (or categorical risk algorithm) is unlikely to be effective compared with prediction algorithms based on diseasespecific and continuous risk markers. It has been shown that the metabolic syndrome, a similar categorical risk criterion to MHO, predicted neither ASCVD nor diabetes satisfactorily [39].

Conclusions People with MHO are not 'healthy' as they are at higher risk of ASCVD, HF and respiratory diseases compared with non-obese people with a normal metabolic profile. As such, weight management could be beneficial to all people with obesity irrespective of metabolic profile. We suggest the term 'MHO' should be avoided in clinical medicine as it is misleading, and different strategies for risk stratification should be explored.

Supplementary Information The online version contains peer-reviewed but unedited supplementary material available at https://doi.org/10.1007/ s00125-021-05484-6.

Acknowledgements We are grateful to UK Biobank participants. This research has been conducted using the UK Biobank Resource under Application Number 7155.

Data availability UK Biobank data can be requested by bona fide researchers for approved projects, including replication, through https:// www.ukbiobank.ac.uk/.

Funding UK Biobank was established by the Wellcome Trust, the Medical Research Council, the Department of Health, the Scottish government and the Northwest Regional Development Agency. It has also had funding from the Welsh Assembly government and the British Heart Foundation.

Authors' relationships and activities NS has received grants and personal fees from Boehringer Ingelheim, and personal fees from Amgen, AstraZeneca, Eli Lilly, Merck Sharp \& Dohme, Novartis, Novo Nordisk, Pfizer and Sanofi outside the submitted work. The remaining authors declare that there are no relationships or activities that might bias, or be perceived to bias, their work.

Contribution statement CC-M, NS, JPP and FKH conceived the idea for the paper. ZZ and FKH conducted the analysis and wrote the first draft. All authors contributed to the interpretation of the findings. All authors critically revised the paper for intellectual content and approved the final version of the manuscript. CC-M, NS, JPP and FKH are guarantors of the work. The corresponding author attests that all listed authors meet authorship criteria and that no others meeting the criteria have been omitted.

Open Access This article is licensed under a Creative Commons Attribution 4.0 International License, which permits use, sharing, adaptation, distribution and reproduction in any medium or format, as long as you give appropriate credit to the original author(s) and the source, provide a link to the Creative Commons licence, and indicate if changes were made. The images or other third party material in this article are included in the article's Creative Commons licence, unless indicated otherwise in a credit line to the material. If material is not included in the article's Creative Commons licence and your intended use is not permitted by statutory regulation or exceeds the permitted use, you will need to obtain permission directly from the copyright holder. To view a copy of this licence, visit http://creativecommons.org/licenses/by/4.0/.

\section{References}

1. Zimmet P, Alberti K, Shaw J (2001) Global and societal implications of the diabetes epidemic. Nature 414(6865):782-787. https:// doi.org/10.1038/414782a

2. Kelly T, Yang W, Chen C-S, Reynolds K, He J (2008) Global burden of obesity in 2005 and projections to 2030. Int J Obes 32(9):1431-1437. https://doi.org/10.1038/ijo.2008.102

3. Kromhout D, Geleijnse JM, Boer JM, Verschuren WM (2009) Body mass index and waist circumference predict both 10-year nonfatal and fatal cardiovascular disease risk: study conducted in 20000 Dutch men and women aged 20-65 years. Eur J Cardiovasc Prev Rehabil 16(6):729-734

4. Hopps E, Noto D, Caimi G, Averna MR (2010) A novel component of the metabolic syndrome: the oxidative stress. Nutr Metab Cardiovasc Dis 20(1):72-77. https://doi.org/10.1016/j.numecd. 2009.06.002

5. Kim SH, Abbasi F, Reaven GM (2004) Impact of degree of obesity on surrogate estimates of insulin resistance. Diabetes Care 27(8): 1998-2002. https://doi.org/10.2337/diacare.27.8.1998

6. Stefan N, Häring H-U, Hu FB, Schulze MB (2013) Metabolically healthy obesity: epidemiology, mechanisms, and clinical implications. Lancet Diabetes Endocrinol 1(2):152-162. https://doi.org/10. 1016/S2213-8587(13)70062-7

7. Hinnouho G-M, Czernichow S, Dugravot A, Batty GD, Kivimaki M, Singh-Manoux A (2013) Metabolically healthy obesity and risk of mortality: does the definition of metabolic health matter? Diabetes Care 36(8):2294-2300. https://doi.org/10.2337/dc121654 
8. Lopez-Garcia E, Guallar-Castillon P, Leon-Muñoz L, RodriguezArtalejo F (2013) Prevalence and determinants of metabolically healthy obesity in Spain. Atherosclerosis 231(1):152-157. https:// doi.org/10.1016/j.atherosclerosis.2013.09.003

9. Zhang M, Tong W, Chen J, Zhang Y, Li S (2014) Metabolically healthy obesity and its associates in Mongolian Chinese adults. Metab Syndr Relat Disord 12(3):185-190. https://doi.org/10. 1089/met.2013.0102

10. Bell JA, Kivimaki M, Hamer M (2014) Metabolically healthy obesity and risk of incident type 2 diabetes: a meta-analysis of prospective cohort studies. Obes Rev 15(6):504-515

11. Hamer M, Stamatakis E (2012) Metabolically healthy obesity and risk of all-cause and cardiovascular disease mortality. J Clin Endocrinol Metab 97(7):2482-2488. https://doi.org/10.1210/jc. 2011-3475

12. Ärnlöv J, Ingelsson E, Sundström J, Lind L (2010) Impact of body mass index and the metabolic syndrome on the risk of cardiovascular disease and death in middle-aged men. Circulation 121(2):230 236. https://doi.org/10.1161/CIRCULATIONAHA.109.887521

13. Twig G, Afek A, Derazne E et al (2014) Diabetes risk among overweight and obese metabolically healthy young adults. Diabetes Care 37(11):2989-2995. https://doi.org/10.2337/dc140869

14. Blüher M (2020) Metabolically healthy obesity. Endocr Rev 41(3): 405-420. https://doi.org/10.1210/endrev/bnaa004

15. Iacobini C, Pugliese G, Blasetti Fantauzzi C, Federici M, Menini S (2019) Metabolically healthy versus metabolically unhealthy obesity. Metab Clin Exp 92:51-60. https://doi.org/10.1016/j.metabol. 2018.11.009

16. Appleton SL, Seaborn CJ, Visvanathan R et al (2013) Diabetes and cardiovascular disease outcomes in the metabolically healthy obese phenotype: a cohort study. Diabetes Care 36(8):2388-2394. https:// doi.org/10.2337/dc12-1971

17. Sudlow C, Gallacher J, Allen N et al (2015) UK Biobank: an open access resource for identifying the causes of a wide range of complex diseases of middle and old age. PLoS Med 12(3): e1001779. https://doi.org/10.1371/journal.pmed.1001779

18. Fry D, Almon R, Moffat S et al (2019) UK Biobank Biomarker Project Companion Document to Accompany Serum Biomarker Data: UK Biobank. Available from https://biobank.ndph.ox.ac.uk/ showcase/showcase/docs/serum_biochemistry.pdf. Accessed 7 Apr 2021

19. van Vliet-Ostaptchouk JV, Nuotio M-L, Slagter SN et al (2014) The prevalence of metabolic syndrome and metabolically healthy obesity in Europe: a collaborative analysis of ten large cohort studies. BMC Endocr Disord 14(1):9

20. Goff DC, Lloyd-Jones DM, Bennett G et al (2014) 2013 ACC/ AHA guideline on the assessment of cardiovascular risk: a report of the American College of Cardiology/American Heart Association Task Force on Practice Guidelines. J Am Coll Cardiol 63(25 Part B):2935-2959

21. Craig CL, Marshall AL, Sjöström M et al (2003) International physical activity questionnaire: 12-country reliability and validity. Med Sci Sports Exerc 35(8):1381-1395. https://doi.org/10.1249/ 01.MSS.0000078924.61453.FB

22. Townsend P, Phillimore P, Beattie A (1987) Health and deprivation. Inequality and the North. Routledge, London

23. Govindarajulu US, Malloy EJ, Ganguli B, Spiegelman D, Eisen EA (2009) The comparison of alternative smoothing methods for fitting non-linear exposure-response relationships with Cox models in a simulation study. Int J Biostat 5(1):Article 2. https://doi.org/10. 2202/1557-4679.1104
24. Tingley D, Yamamoto T, Hirose K, Keele L, Imai K (2014) Mediation: R package for causal mediation analysis. J Stat Softw https://doi.org/10.18637/jss.v059.i05

25. Collins R (2012) What makes UK Biobank special? Lancet 379(9822):1173-1174. https://doi.org/10.1016/S0140-6736(12) 60404-8

26. Batty GD, Gale CR, Kivimäki M, Deary IJ, Bell S (2020) Comparison of risk factor associations in UK Biobank against representative, general population based studies with conventional response rates: prospective cohort study and individual participant meta-analysis. BMJ 368:m131. https://doi.org/10.1136/bmj.m131

27. Di Angelantonio E, Gao P, Khan H et al (2014) Glycated hemoglobin measurement and prediction of cardiovascular disease. JAMA 311(12):1225-1233. https://doi.org/10.1001/jama.2014.1873

28. Korduner J, Bachus E, Jujic A, Magnusson M, Nilsson PM (2019) Metabolically healthy obesity (MHO) in the Malmö diet cancer study-epidemiology and prospective risks. Obes Res Clin Pract 13(6):548-554. https://doi.org/10.1016/j.orcp.2019.10.002

29. Nilsson PM, Korduner J, Magnusson M (2020) Metabolically healthy obesity (MHO) - new research directions for personalised medicine in cardiovascular prevention. Curr Hypertens Rep 22(2): $1-5$

30. Lassale C, Tzoulaki I, Moons KGM et al (2018) Separate and combined associations of obesity and metabolic health with coronary heart disease: a pan-European case-cohort analysis. Eur Heart J 39(5):397-406. https://doi.org/10.1093/eurheartj/ehx448

31. Sattar N, McGuire DK (2018) Pathways to cardiorenal complications in type 2 diabetes mellitus: a need to rethink. Circulation 138(1):7-9. https://doi.org/10.1161/CIRCULATIONAHA.118. 035083

32. Rosengren A, Åberg M, Robertson J et al (2017) Body weight in adolescence and long-term risk of early heart failure in adulthood among men in Sweden. Eur Heart J 38(24):1926-1933. https://doi. org/10.1093/eurheartj/ehw221

33. Poulain M (2006) The effect of obesity on chronic respiratory diseases: pathophysiology and therapeutic strategies. Can Med Assoc J 174(9):1293-1299. https://doi.org/10.1503/cmaj.051299

34. Franssen FME, O’Donnell DE, Goossens GH, Blaak EE, Schols AMWJ (2008) Obesity and the lung: 5. Obesity and COPD. Thorax 63(12):1110-1117. https://doi.org/10.1136/thx.2007.086827

35. Soriano JB, Kendrick PJ, Paulson KR et al (2020) Prevalence and attributable health burden of chronic respiratory diseases, 1990 2017: a systematic analysis for the Global Burden of Disease Study 2017. Lancet Respir Med 8(6):585-596. https://doi.org/10. 1016/S2213-2600(20)30105-3

36. Igel LI, Kumar RB, Saunders KH, Aronne LJ (2017) Practical use of pharmacotherapy for obesity. Gastroenterology 152(7):17651779. https://doi.org/10.1053/j.gastro.2016.12.049

37. Wilding JP, Batterham RL, Calanna S et al (2021) Once-weekly semaglutide in adults with overweight or obesity. N Engl J Med 384(11):989-1002. https://doi.org/10.1056/NEJMoa2032183

38. Busetto L, Dicker D, Azran C et al (2017) Practical recommendations of the obesity management task force of the European Association for the Study of obesity for the post-bariatric surgery medical management. Obes Facts 10(6):597-632

39. Sattar N, McConnachie A, Shaper AG et al (2008) Can metabolic syndrome usefully predict cardiovascular disease and diabetes? Outcome data from two prospective studies. Lancet 371(9628): 1927-1935. https://doi.org/10.1016/S0140-6736(08)60602-9

Publisher's note Springer Nature remains neutral with regard to jurisdictional claims in published maps and institutional affiliations. 